\title{
UN'ANTICA RIFLESSIONE SULLE QUESTIONI DI GENERE. LE RICADUTE PEDAGOGICHE DE LA CITTÀ DELLE DAME
}

\author{
AN OLD THOUGHT ABOUT GENDER ISSUES. THE PEDAGOGICAL RELAPSES \\ OF THE BOOK OF THE CITY OF LADIES
}

Barbara De Serio

Universidad de Foggia

\section{RIASSUNTO:}

Vengono percorse le fasi principali della riflessione medievale sulla questione femminile analizzando il contenuto delle prime dispute di genere per la tutela dei diritti delle donne attraverso La Città delle Dame, di Christine de Pizan. Partendo dalla lettura della sua reazione polemica nei confronti di una tradizione misogina, caratteristica degli scrittori medievali, viene approfondito un nuovo modello di donna proposto dall'autrice che per prima ha ipotizzato la formazionedi un'alleanza di genere e di una comunità femminile.

\section{PAROLE CHIAVE:}

Genere, pari opportunità, donne e progresso sociale.

\section{ABSTRACT:}

This paper traces the medieval reflection on women's issues and analyses the first gender identity disputes to protect the rights of women through reading of The Book of the City of Ladies, by Christine de Pizan. From the reading of her polemic reaction against the misogynist tradition, that appears in many medieval works, it is analysed a new female model proposed by the author, who first suggested an alliance of gender and a women community.

\section{KEY wORD:}

Gender, equal opportunities, women and social progress. 


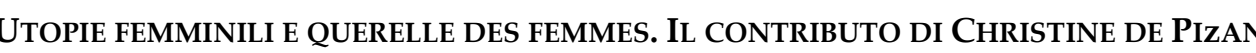

Era il 1404 quando Christine de Pizan, scrittrice francese di origini italiane, cominciò a scrivere La Città delle Dame, un romanzo che le valse il titolo di scrittrice europea di professione e che fece di lei la precorritrice dei movimenti culturali contrari alla misoginia e delle dispute di genere per la tutela dei diritti delle donne, dei quali si fece portavoce in tutte le sue opere. In virtù delle sue idee emancipatrici a favore delle donne, espressione di una genealogia femminile orientata a superare le principali cause dell'ostilità fra i due sessi, Christine de Pizan viene tutt'oggi annoverata tra le voci femminili più autorevoli del Medioevo, antesignane delle prime riflessioni sulla questione femminile che si sarebbe diffusa qualche decennio dopo e che a distanza di cinque secoli sarebbe sfociata nei primi movimenti femministi. Il suo nome compare, infatti, tra quelli delle più attive esponenti della disputa letteraria sulla superiorità di uno dei due sessi sull'altro, nota come querelle des femmes ${ }^{1}$.

Lo scopo degli intellettuali che parteciparono alla disputa - e che nei loro scritti non perdevano occasione per denigrare le donne, la cui ignoranza veniva considerata la principale causa della degenerazione dei costumi e della società era quello di mettere in risalto la leggerezza e la superficialità del comportamento femminile. Contro tali stereotipi si schierò Christine de Pizan ne La Città delle Dame, che può essere considerata la reazione polemica alle maldicenze sulle donne, ovvero ad una tradizione religiosa molto forte che invitava a sfuggire ogni occasione di peccato nonché ad una cultura misogina che sembrava costituire l'unico strumento per combattere la seduzione incarnata dal genere femminile. La scelta di scrivere un libro sulle donne nacque dal bisogno di comprendere lecause per cui tanti uomini diversi tra loro per condizione [...] erano stati ed erano ancora così propensi a dire e a scrivere nei loro trattati tante diavolerie e maldicenze sulle donne e la loro condizione. $[\ldots]$ in ogni trattato filosofi e poeti, predicatori $[\ldots]$ sembrano tutti parlare con la stessa bocca, tutti d'accordo nella medesima conclusione, che il comportamento delle donne è incline a ogni tipo di vizio. [...] per quanto a lungo e profondamente esaminassi la questione - conclude la scrittrice - non riuscivoa riconoscere né ad ammettere il fondamento di questi giudizi contro la natura e il comportamento femminile (de Pizan 1404-1405/2010:43)

Eppure la forza dei condizionamenti sociali e l'assenza di una tradizione letteraria femminile in grado di confutarli la indussero «a pensare male delle donne» perché uomini «di così grande intelligenza» non avrebbero mai potuto rivolgere erroneamente parole di biasimo nei confronti del genere femminile:

1 La figura di Christine de Pizan è ancora più significativa poiché il suo pensiero rappresenta Revista Internacional de Culturas y Literaturas, abril 2012 ISSN: $1885-3625$ benché il mio intelletto nella sua semplicità e ignoranza non sapesse riconoscere i grandi difetti miei come delle altre donne, doveva essere veramente così. Era in questo modo che mi affidavo più ai giudizi altrui che a ciò che io sentivo e sapevo [...]. Alla fine decisi che Dio aveva fatto una cosa ben vile quando creò la donna meravigliandomi che un artigiano così degno avesse realizzato un'opera tanto abominevole, ricettacolo [...] di tutti i mali e di tutti i vizi. [...] disprezzavo me stessa e tutto il sesso femminile, cor 1404-1405/2010:43-47).

È a questo punto che nella sua vita appaiono Ragione, Rettitudine e Giustizia, ${ }^{2}$ tre dame «dal portamento maestoso», avvolte da «un fascio di luce», secondo una tradizione mistica allora molto diffusa, e capaci di rischiarare l'ambiente «come un raggio di sole» (de Pizan 1404-1405/2010, p. 43). La metafora è chiara, soprattutto perché a parlare per prima è Ragione, espressione figurata del pensiero flessibile, in grado di superare le false credenze e di costruire le fondamenta di un nuovo sapere, antidogmatico e plurale. La dama interviene per sgomberare la mente della scrittrice dai vincoli culturali che non le consentono di pensare autonomamente, di esprimere il proprio punto di vista e di esercitare il potere della ragione, unico strumento per demolire i pregiudizi: «vorremmo toglierti dall'ignoranza, che ti acceca tanto da farti dimenticare ciò che conosci con certezza, per credere a qualcosa che sai, vedi e conosci solo per le numerose opinioni altrui» (de Pizan 1404-1405/2010, p. 47). Per questo motivo la esorta a recuperare l'autostima necessaria per riconoscere le potenzialità e le risorse di cui è in possesso il genere femminile e a riscattarsi da un luogo comune che voleva che la donna fosse per natura volubile e quindi oggetto di offesa e maltrattamento da parte del genere maschile. Infine la invita a riflettere sulla quantità di scritti sulle donne, che dimostrano come «sono le cose migliori ad essere le più dibattute e le più discusse» (de Pizan 1404-1405/2010, p. 49).

Con la consapevolezza che occorre «rifuggire e disprezzare la menzogna» la scrittrice racconta dunque di aver ascoltato piacevolmente il discorso delle tre dame e di aver accolto l'invito a «prendere la zappa dell'intelligenza, a scavare con forza un grande fossato e a portare via la terra» (de Pizan 1404-1405/2010), ovvero a liberare la cultura dai preconcetti per renderla fertile e predisposta ad accogliere ogni tipo di sapere, compreso quello femminile.

Con l'ago e con la penna. Come progettare un nuovo modello di donna

Il carattere innovativo del suo pensiero politico ed etico è connesso all'educazione familiare ricevuta, che ricorre spesso nelle sue opere, nelle quali la scrittrice racconta di essere stata fortunata innanzitutto per aver ottenuto la possibilita di studiare, che in quel periodo veniva negata alle bambine, e in secondo luogo 
Un'antica riflessione sulle questini di genere
2 La scelta delle tre dame protagoniste del romanzo non è casuale: Ragione, Rettitudine e Giustizia rappresentano le qualità femminili necessarie per promuovere l'uguaglianza, per valorizzare la rappresentano le qualita femminili necessarie per promuovere l'uguaglianza, per valorizar
differenza e per costruire un rapporto di mediazione, di equilibrio e di stabilita tra i generi. 
per aver potuto godere del confronto con un modello genitoriale comunicativo, con particolare riferimento alla figura paterna, che l'ha progressivamente indotta a maturare una visione più emancipata del genere femminile, svincolata dalle pressioni sociali e dalle categorizzazioni culturali connesse alla differenziazione dei ruoli sessuali. A differenza della madre, che la «voleva occupata con ago e filo nelle attività consuete delle donne e che per questo motivo fu per lei $\mathrm{y}^{\prime}$ os e filo nè̀ ativita col «t ostacolo piu grande allo studio e all approfondimento delle scienze», il padre, medico e astrologo, anon pensava ceto he le donne fossero meno capaci di imparare le scienze» (de Pizan 1404-1405/2010, p. 317) e questo suo atteggiamento fu per lei uno stimolo per riflettere sul destino comunemente riservato alle bambine e sulle qualità femminili che gli spazi tradizionalmente riservati loro oscuravano, ovvero sulle capacità che occorreva promuovere per emancipare la donna dagli stereotipi

di genere che inibivano le sue potenzialità

A causare l'inferiorità cognitiva della donna non era tanto l'impossibilità $\mathrm{d}$ partecipare attivamente alla vita sociale, relegata com'era tra le mura domestiche, che costituivano l'unico spazio possibile per la donna di sani costumi morali, quanto piuttosto il diritto ad un sapere limitato, ovvero l'assenza, tra quelle mura, di qualcuno che potesse prendersi cura della sua istruzione, che le veniva negata per il semplice fatto di appartenere ad un genere intellettualmente debole o forse - come lei stessa scrive - perché gli uomini si sarebbero sentiti minacciati dal potere di una donna istruita. Tale riflessione la indusse ad impostare un rapporto coniugale differente e meno convenzionale, più rispettoso della parità sessuale e della comunicazione tra i generi e più fiducioso nel valore della complementarità e del confronto costruttivo: «Dio -scrive ne La Città delle Dame- ha stabilito che l'uomo e la donna Lo servano in maniera diversa e che si aiutino e confortino reciprocamente, l'uno compagno dell'altra, ognuno in ciò che gli è destinato e ha dato a ogni sesso una natura e inclinazioni diverse, secondo i compiti rispettivi» (de Pizan 1404-1405/2010, p. 93). Anche in questo Christine de Pizan fu rivoluzionaria, poiché l'immagine di famiglia che emerge dai suoi scritti focalizza l'attenzione sulla libera scelta del coniuge e sull'unione coniugale basata sul coinvolgimento affettivo, anticipando di qualche secolo la comparsa del modello coniugale intimo che caratterizzerà il sistema familiare a partire dalla fine dall'età moderna

La Città delle Dame. Un luogo per ripercorrere la storia di genere $\mathrm{Ne}$

La Città delle Dame emerge in modo evidente la forza emancipatrice dell'istruzione,

tanto che il romanzo è stato definito il «luogo utopico del trionfo dell'onore femminile» (Giallongo1995:222), lo spazio pubblico di trasmissione dei saperi delle donne, che hanno fatto della loro creatività uno strumento di trasformazione culturale e di rinnovamento morale, un dispositivo per decostruire i pregiudizi sessisti, di chiara derivazione androcentrica, e per costruire un nuovo modello culturale, capace di includere l'originalità del sapere femminile accanto a quello maschile. Un sapere che Christine de Pizan ha recuperato proprio a partire dalla valorizzazione della dimensione dell'emotività e dalla «naturale predisposizione al pianto», espressione di «grande saggezza» delle donne e manifestazione «della dolcezza della loro condizione femminile» e della loro sublime capacità di cura (de Pizan,1404-1405/2010).

Christine de Pizan vede nella donna, e nella sua capacità critica e creativa, lo strumento vitale e propositivo per promuovere un incontro tra le differenze e per ristabilire un equilibrio tra i generi -maschile e femminile- nell'ottica di un'autentica cultura della pace. Tutto questo a partire dalla sua naturale disponibilità cognitiva a riconoscere l'alterità e ad accoglierla come occasione di arricchimento personale.

Per questo motivo La Città delle Dame è stato definito il romanzo della «storia al contrario» (Giallongo, 2005), una «riscrittura della tradizione» (Caraffi 2010) in grado di esortare le donne a riconoscere le proprie potenzialità. Christine de Pizan vi presenta diversi modelli di donne del passato che si sono distinte come maestre di civiltà per una naturale inclinazione all'esercizio dei pubblici poteri e per una altrettanto naturale tendenza a mediare i conflitti e a mettere il proprio sapere al servizio del sviluppo sociale e della rigenerazione dell'umanità.

Per certi versi la città progettata nel romanzo sembra anticipare la metafora della stanza tutta per sé di Virginia Woolf, nella quale la donna è riuscita a ritagliarsi uno spazio personale per entrare in rapporto con la realtà interiore e per ritrovare in questo spazio sommerso la propria femminilità. Sede di costruzione della memoria individuale, spazio di consapevolezza di sé, in cui rivendicare il proprio bisogno intellettuale e il proprio diritto all'istruzione, la città delle dame di Christine de Pizan rappresenta infatti il luogo più adatto per salvaguardare il pensiero femminile dalle ingerenze maschili. Ma a differenza di Virginia Woolf Christine de Pizan crede nell'unione femminile e ritiene che la partecipazione attiva -e non l'isolamento- costituisce la forza per combattere lo stato di emarginazione delle donne, accanto all'interazione e allo scambio costruttivo con altre donne. La città delle dame non rappresenta, dunque, una stanza tutta per sé, ma uno spazio per tutte le donne, un luogo privato in cui donne appartenenti a diversi ceti sociali possono incontrarsi per condividere le proprie conoscenze, che in questa città fortificata, retta dalla forza e dalla nobiltà dei saperi femminili, possono proteggere e conservare inalterate, affidando alla scrittura i più intimi segreti.

Le tre dame, come pure le donne che alla fine del romanzo Christine de Pizan viene invitata a chiamare perché possano abitare la città da lei istituita, rappresentano dunque un modello di comunità femminile ed esprimono quella solidarietà e quell'alleanza di genere che è tipica del sapere delle donne e che costituisce spesso l'unico strumento per combattere le ingiustizie e per moralizzare il pensiero maschile (Caraffi 2003:19$31)$.

Metamorfosi e bisogno di riconoscimento 
Le ricadute pedagogiche del romanzo vanno rintracciate nella sua capacitàdi diventare un dispositivo di potere per le donne, uno strumento per educare all'empowerment (Giallongo 2005), per restituire alle donne un buon livello di autostima e di fiducia in se stesse, per guidarle a riconoscere le potenzialità di cui sonoin possesso, per incoraggiarle ad esercitare un potere sul proprio sapere, a prendere decisioni autonome e ad utilizzare il proprio potenziale creativo per se stesse e per glialtri. Più precisamente, il romanzo esorta le donne ad individuare nel proprio sapere le condizioni per influenzare positivamente il proprio contesto di appartenenza e per ridurre o eliminare le cause della propria povertà culturale e del deterioramento sociale dell'ambiente in cui vivono, spesso eccessivamente custodito e restrittivo. Diventare empowered, sembra dire Christine de Pizan nell'introduzione e nella conclusione del romanzo, non significa soltanto emanciparsi da una condizione di minorità fisica e cognitiva rispetto al genere maschile ${ }^{3}$, ma soprattutto riconoscere che ogni possibilità di mediazione dipende da una scelta personale, dalla propria capacità di acquisire il coraggio necessario per valorizzare i poteri del quotidiano, dalla propria volontà di mettere ordine al disordine e di ridare un senso alle cose, dalla propria capacità di essere resilienti. Lei stessa lo è stata quando ha accettato di assumere la responsabilità di guidare da sola le famiglie che il marito e il padre, morendo, le avevano affidato e quando ha imparato a riconoscere che lunghi periodi di dolore possono rafforzare l'animo umano e renderlo appunto resiliente. Questo disvelamento della propria forza interiore è il primo passo verso il superamento delle emozioni negative, che inibiscono e creano insicurezza e che per questo motivo sono spesso la causa della dipendenza della donna.

È dunque la percezione della propria femminilità nonché la consapevolezza dell'appartenenza al genere femminile a rendere resilienti e non l'affiliazione al genere maschile e all'aggressività che lo caratterizza e che viene spesso confusa con il coraggio e la determinazione. Un errore che lei stessa racconta di aver commesso dopo la morte dei suoi familiari, ovvero nel periodo in cui fu vittima di una forte disperazione che la indusse a vivere come un'ulteriore punizione del destino l'essere nata donna. È opportuno a tal proposito ricordare la metamorfosi che Christine de Pizan descrive ne L'Avision (de Pizan 1405/1932), dove racconta la sua trasformazione in un uomo, che può però essere letta come la scelta coraggiosa di una donna che vuole ribellarsi ad una condizione di inferiorità e che riesce a realizzare un modello di femminilità innovativo a partire dalla scelta di esercitare la professione di scrittrice, tradizionalmente riservata agli uomini. Tale decisione, inizialmente dettata da necessità di carattere economico, rivelò presto una pretesa più alta, connessa al bisogno di dimostrare che anche le donne possiedono qualità intellettuali di grande spessore -oltre ad un profondo senso

3 «Un corpo grande e forte non garantisce un virtuoso e grande coraggio $[\ldots]$ che risiede nell' interiorità e non nella forza del corpo e delle membra» (de Pizan 1404-1405/ 2010, p. 103). di civiltà- che utilizzano in ambito domestico, ma che se necessario vengono messe a disposizione della società e del suo progresso ${ }^{4}$. Questo èil suo modello di emancipazione femminile: una donna che svolge un lavoro maschile senza però disprezzare le attività femminili, alle quali anzi si applica volentieri. Una donna autonoma e indipendente, finalmente capace di pensare a se stessa e quindi di sottrarsi a tutte le forme di controllo comunemente utilizzate dal genere maschile.

Con evidenti rimandi alla sua esperienza personale e in linea con le teorie medievali sulla condizione femminile, che vedevano nella figura della sposa il modello di femminilità per eccellenza e che paradossalmente consideravano il vincolo matrimoniale l'unica condizione di libertà per la donna, Christine de Pizan sembra individuare nell'immagine della donna vedova, sposa matura, fedele al suo ruolo di moglie e sola non per volontà personale, il più autentico modello di donna indipendente. La Città delle Dame è dunque un inno alla femminilità poiché le donne, dotate di notevole saggezza, di elevata sensibilità e di grande forza d'animo, vengono presentate come modelli di moralità. Ed è anche questo il motivo per cui a progettare la costruzione della città delle dame sono tre donne: non solo perché più capaci di comprendere il bisogno di emancipazione femminile, ma anche perché solo le donne hanno il potere di costruire dove gli uomini distruggono e di agire con quella costanza che è tipica di chi sa attendere con pazienza e di chi sa cogliere proprio nell'attesa il tempo dell'azione.

\section{RIFERIMENTI BIBLIOGRAFICI}

Bock, G., Le donne nella storia europea. Dal Medioevo ai nostri giorni, Roma-Bari, Laterza, 2006. Caraffi, P. (a cura di), Christine de Pizan. Una città per sé, Roma, Carocci, 2003.

De Pizan, C., La città delle dame (1404-1405), Roma, Carocci, 2010.

Giallongo, A., L'avventura dello sguardo. Educazione e comunicazione visiva nel Medioevo, Bari, Dedalo, 1995.

----, Il bambino medievale, Bari, Dedalo, 1997.

---- (a cura di), Donne di palazzo nelle corti europee. Tracce e forme di potere dall'età moderna, Milano, Unicopli, 2005.

Klapish Zuber, C., (a cura di), Storia delle donne in Occidente. Il Medioevo, Roma-Bari, Laterza, 2009.

Woolf, V., Saggi, prose, racconti, Milano, Mondadori, 2004.

4 L'utilizzo della penna da parte di una donna era considerato un atto trasgressivo poiché espressione di un potere intellettuale e di un controllo personale sul proprio pensiero (Cerquiglini Toulet, 2003, pp. 71-85). 